\title{
sciendo
}

\section{Defining Metabolic Syndrome: Which Kind of Causality, if any, is Required?}

\author{
Margherita Benzi \\ University of Eastern Piedmont \\ BIBLID [0873-626X (2017) 47; pp. 553-580] \\ DOI: 10.1515/disp-2017-0018
}

\begin{abstract}
The definition of metabolic syndrome (MetS) has been, and still is, extremely controversial. My purpose is not to give a solution to the associated debate but to argue that the controversy is at least partially due to the different 'causal content' of the various definitions: their theoretical validity and practical utility can be evaluated by reconstructing or making explicit the underlying causal structure. I will therefore propose to distinguish the alternative definitions according to the kinds of causal content they carry: (1) definitions grounded on associations, (2) definitions presupposing a causal model built upon statistical associations, and (3) definitions grounded on underlying mechanisms. I suggest that analysing definitions according to their causal content can be helpful in evaluating alternative definitions of some diseases. I want to show how the controversy over MetS suggests a distinction among three kinds of definitions based on how explicitly they characterise the syndrome in causal terms, and on the type of causality involved. I will call 'type 1 definitions' those definitions that are purely associative; 'type 2 definitions' the definitions based on statistical associations, plus generic medical and causal knowledge; and 'type 3 definitions' the definitions based on (hypotheses about) mechanisms. These kinds of definitions, although different, can be related to each other. A definition with more specific causal content may be useful in the evaluation of definitions characterised by a lower degree of causal specificity. Moreover, the identification of the type of causality involved is of help to constitute a good criterion for choosing among different definitions of a pathological entity.

In section (1) I introduce the controversy about MetS, in section (2) I propose some remarks about medical definitions and their 'causal import', and in section (3) I suggest that the different attitudes towards the definition of MetS are relevant to evaluate their explicative power.
\end{abstract}




\section{Keywords}

Metabolic syndrome, medical definitions, causal models, mechanistic property clusters, natural kinds

\section{A short historical survey on metabolic syndrome}

The debate on the notion of metabolic syndrome (MetS), a now ubiquitous topic in both scientific literature and the media, began in late 1980s and reached a peak in the first decade of this century. Given that the topic of MetS is still highly debated, I will restrict the short historical survey to the period in which the conflict among definitions is particularly strong and methodologically explicit. This narrower historical focus helps to show that the controversy over the definitions of MetS is interwoven with some classical problems in the philosophy of medicine, mainly whether definitions must have a causal import or how to understand causality in this context.

\subsection{The rise of metabolic syndrome}

MetS has long been known as a constellation of factors that put individuals at risk of developing two relevant pathologies: type 2 diabetes (T2D) and cardiovascular disease (CVD). In addition, MetS has been recognised as an important risk factor in other pathologies, such as non-alcoholic fatty liver disease, polycystic ovary syndrome, obstructive sleep apnea, sexual dysfunction, and some kinds of cancer, particularly colon, prostate, and breast cancers. According to the International Diabetes Federation (IDF), a quarter of the world's adults have MetS (see www. idf.org/metabolic-syndrome). Not only is MetS so often in the public eye that the media describe it as the $21^{\text {st }}$-century plague, but — as a rapid search on PubMed can easily testify - it is also the source of an impressive number of scientific publications in the biomedical and health areas.

Despite its (sinister) fame, in the first decade of this century, MetS has been one of the most controversial notions of biomedical ontology. On the one hand, there is a shared agreement on the existence of MetS (or of some cognate notion); on the other hand, there is almost no agreement on its constitutive elements, its structure, how to diagnose it, who exactly suffers from it, and its causal role relative 
to its presumed complications. This and the following section contain an overview of the history of MetS; the focus is on the period 1988-2010, when the temperature of the debate reached its peak.

In 1761 the Italian anatomist G. B. Morgagni described in his book De sedibus et causis morborum per anatomen indagati an association of abdominal obesity and hypertension, hyperuricemia, atherosclerosis, and obstructive sleep apnea (see Enzi et al. 2003). After the First World War, two Austrian physicians, K. Hitzenberger and M. Richter-Quittner (1921), published their laboratory observations, which suggested a link between hypertension and diabetes mellitus. Other results were published by the Spanish doctor G. Marañon (1922) and by the Swedish physician E. Kylin (1921, 1923); the latter described a clustering of hypertension, hyperglycaemia, and gout. In 1947, J. Vague published his observations on the so-called android, or male, or apple-shaped obesity, noticing that this form of obesity is associated with the metabolic abnormalities often seen with T2D and CVD. The term 'metabolic syndrome' was introduced in 1975 by Haller and Hanefeld while studying risk factors of atherosclerosis. Kissebah et al. (1982) reported that upper-body obesity in women offered an important prognostic marker for glucose intolerance, hyperinsulinemia, and hypertriglyceridemia, and Fujioka et al. (1987) distinguished between visceral fat obesity and subcutaneous fat obesity.

The contributions quoted above are generally considered as the prehistory of MetS. Most authors set the official date of the birth of the syndrome in 1988, when an American endocrinologist, Gerald Reaven, proposed to call 'Syndrome X' a list of phenotypic abnormalities, whose clustering appeared to be predictive of T2D (Reaven 1988). The components of Syndrome $X$ were the following:

(a) Resistance to insulin-stimulated glucose uptake;

(b) Impaired glucose tolerance;

(c) Hyperinsulinemia;

(d) Increased VLDL (= very-low-density-lipoprotein) triglycerides;

(e) Decreased HDL (= high-density lipoprotein) cholesterol; 
and

(f) Hypertension.

In his paper, Reaven suggests that insulin resistance ${ }^{1}$ (a) plays a fundamental role in the pathogenesis of coronary artery disease (CAD) through various associations with (b), (c), (d), (e), and (f), where (f) can in turn causally depend on (a) and (c). The hypothesised causal relations among the variables are tested by experimental manipulations of rats, or by observations of patients. For instance, Reaven (1988: 602) provides experimental evidence that the association of insulin resistance (a), glucose intolerance (b), and hyperinsulinemia (c) is characteristic of a certain patients with hypertension (f); the dependency, in these patients, of hypertension on (a), (b), and (c) is supported by the observation that 'these abnormalities of glucose and insulin metabolism do not necessarily improve when hypertension is controlled by commonly used pharmacological approaches to lower blood pressure'. Further experimental evidence is provided by the fact that 'hypertension can be produced in normal rats by an experimental manipulation known to induce insulin resistance and hyperinsulinemia' (Reaven 1988: 1604). Insulin resistance is the precipitator of Syndrome X:

The common feature of the proposed Syndrome is insulin resistance, and all other changes are likely to be secondary on this basic abnormality. All five of the proposed consequences of insulin resistance have been shown to increase the risk of $\mathrm{CAD}$, and the fact that all of them may not necessarily be seen in the same individual should not minimize their importance (Reaven 1988: 1606).

Since the introduction of Syndrome X, there has been considerable new information on the role of insulin resistance in increasing the risk of polycystic ovary syndrome, non-alcoholic fatty liver disease, certain forms of cancer, and sleep apnea. These findings induced Reaven to expand the list of abnormalities linked to insulin resistance and to call the new list 'insulin resistance syndrome' or 'IR syndrome' (Reaven 2004).

\footnotetext{
${ }^{1}$ Insulin resistance is the inability to regulate the action of insulin on adipose tissue and muscle.
} 


\subsection{The controversy}

After Reaven's 1988 contribution, a plethora of definitions was provided (see Lam and LeRoith 2015). In the same year, the World Health Organization (WHO) produced a working definition of MetS. The definition required having impaired glucose tolerance, impaired fasting glucose or diabetes mellitus, and/or insulin resistance together with two or more additional components. The additional components were microalbuminuria, hypertension, high triglycerides, low HDL, and central obesity (defined by means of either body mass index or waist-to-hip ratio). The definition also included a quantitative specification of the last four components.

Due to various critiques, some of which were of a practical nature, the European Group for the Study of Insulin Resistance (EGIR) modified the WHO definition in order to simplify the diagnosis (Balkau and Charles 1999); the new definition included obesity but not albuminuria. Additional definitions were provided by the National Cholesterol Education Program (NCEP), which elaborated a version called ATP (Adult Treatment Panel) III (ATP III 2001). In 2005 the IDF consensus definition (Alberti et al. 2005, IDF 2005) included central obesity and excluded insulin resistance, while a revision of the NCEP definition by the American Heart Association (AHA) and the National Heart, Lung, and Blood Institution (NHLBI) did not include abdominal obesity as a necessary component of MetS.

The definitions continued to proliferate, with many 'regional' versions, leading many authors to question the very existence of the syndrome (see Mitka 2005). Then in 2009 a large group of medical institutions elaborated a common 'Worldwide Consensus Statement' (Alberti et al. 2009). The statement endorsed the AHA/NHLBI criteria, but the cut-off points of waist circumference were not determined for any particular ethnic group (see Oda 2012).

The overabundance of definitions by health institutions was criticized (but also involuntary stimulated) by the American Diabetes Association (ADA) and the European Association for the Study of Diabetes (EASD), which published a joint statement that concluded that MetS had been imprecisely defined. The statement advised physicians not to label patients with the term 'metabolic syndrome' in order to avoid creating the false impression that the syndrome 
denotes a greater risk than its components or that the underlying pathophysiology is clear (Kahn et al. 2005).

Unsurprisingly, Reaven maintained a position close to that of the ADA and EASD, and in many writings he carefully distinguished his 'Syndrome X' from 'metabolic syndrome'. Reaven opposed the term 'metabolic syndrome' because many factors of Syndrome X, unlike most definitions of MetS, had a clear pathophysiological grounding. ${ }^{2}$ Within Reaven's framework, obesity is not included among the components of the syndrome, because, despite its recognised association with T2D and CVD, it does not play any recognisable causal role. Syndrome $\mathrm{X}$ and the subsequent IR syndrome are grounded on a mechanistic view where insulin resistance is a common cause of the disease, whereas the definitions of MetS given by the various panellists are grounded on statistical associations and are pragmatically conceived as a tool to implement a healthy lifestyle (Reaven 2004: 297).

Reaven criticised the panellists' definitions of MetS in many articles, one of which had a transparently critical title ('The Metabolic Syndrome: Requiescat in Pace', 2005). In a 2006 article, in which the diagnostic value of MetS is further criticised, Reaven once again stigmatises what he considers the main weak point of the panellists' definitions of MetS: their inability to select a common cause for the clustering factors composing the syndrome. But was Reaven right in

\footnotetext{
${ }^{2}$ In Reaven's own words: 'Sensitivity to insulin-mediated glucose disposal varies widely in the population at large ... . When insulin-resistant individuals cannot maintain the degree of hyperinsulinemia needed to overcome the insulin resistance, type 2 diabetes develops ... . Most individuals are able to sustain the level of compensatory hyperinsulinemia needed to maintain normal or near normal glucose tolerance, however. Unfortunately, this philanthropic effort on the part of the pancreatic beta cell is a mixed blessing. Although the compensatory hyperinsulinemia prevents the development of frank hyperglycaemia, insulin-resistant/hyperinsulinemic individuals are at greatly increased risk of having some degree of glucose intolerance, a high plasma triglyceride and low high-density lipoprotein cholesterol (HDL-C) concentration, and essential hypertension .... . In $1988 \ldots$, it was proposed that individuals who displayed this cluster of abnormalities associated with insulin resistance/compensatory hyperinsulinemia were at significantly increased risk of cardiovascular disease (CVD). Because the importance of insulin resistance and associated abnormalities as CVD risk factors was not widely appreciated at that time, the cluster of related abnormalities was subsumed under the rubric of syndrome X' (Reaven 2004: 283).
} 
criticising the notion of MetS on these grounds? why, and to what extent, should considerations about causality mix with taxonomical questions? I will examine the matter in the following sections.

\section{The causal content of the definition}

In this and the following sections, I consider three possible approaches to the definition of MetS, characterised by different degrees of causality and different conceptions of causality. Here I call 'type 1 definitions' those definitions of MetS that are formulated and justified independently of causal considerations and are grounded mainly on associations of properties. 'Type 2 definitions' find their justification in being associated to a causal model grounded on statistical data. 'Type 3 definitions' reflect a different approach to causality, since they require an associated pathophysiological mechanism that links the various components of the syndrome. In the following sections, I will specify the utility, aims, and functions of the three kinds of definitions, and I will analyze their epistemic benefits.

\subsection{Causally neutral definitions (type 1)}

In what follows, I will consider the causal content of three definitions of MetS, given respectively by ATP III (2001), IDF (2005), and WHO (Alberti and Zimmet 1988). I will then compare the three definitions with the 'parallel' definition of Syndrome X by Reaven.

ATP III defined MetS as any combination of at least three out of five factors (briefly, elevated waist circumference, elevated triglycerides, reduced HDL cholesterol, elevated blood pressure, and elevated fasting glucose level). As the criteria are not subdivided into 'necessary' and 'accessory', ATP III is considered the most 'democratic' of the definitions of MetS. This character can be explained by the fact that the proponents of the definition are sceptical about the possibility of discovering a unique common cause for the clustering of the factors. In a conjoined AHA/NHLBI Scientific Statement on the diagnosis and management of MetS, Grundy et al. state:

${ }^{3}$ See Reaven 2006: 1239. 
At present, it is not clear whether the metabolic syndrome has a single cause, and it appears that it can be precipitated by multiple underlying risk factors. The most important of these underlying risk factors are abdominal obesity and insulin resistance. Other associated conditions include physical inactivity, aging, hormonal imbalance, and genetic or ethnic disposition (Grundy et al. 2006: 1).

Unsurprisingly, given the heterogeneity of the risk factors and associated conditions, in the conclusions they remark that 'it is recognized that the metabolic syndrome is a complex disorder, with no single factor as the cause' (Grundy et al. 2006: 5).

The WHO and the IDF definitions take different approaches in the selection of an essential criterion, even if its causal role is not completely clear. The WHO definition requires that the patient has one of the following conditions: diabetes mellitus, impaired glucose tolerance, impaired fasting glucose, or insulin resistance. Additionally, the patient must exhibit two of the following conditions: elevate waist-to-hip ratio, elevate triglycerides, and microalbuminuria. In this definition, the role of insulin resistance as a possible underlying common cause is recognised, as the factors in the first category are more important and provide evidence in favour of insulin resistance, but there is no indication of a causal mechanism. ${ }^{4}$

The IDF definition also attributes different importance to factors, but here the fundamental criterion is abdominal obesity; in addition, two of the following factors are required: elevated triglycerides, reduced HDL cholesterol, high blood pressure, and high fasting plasma glucose. In the definitions given by panellists, we can find a descriptive approach, where there is no specific search for the causal role carried out by each factor. Basically, these definitions state that exhibiting a certain number of properties recognised as constitutive elements of the 'syndrome' is associated with an increased risk of certain pathologies.

Federspil et al. (2006) were the first to stress the philosophical interest of the controversy. They remark that a long tradition in

4 'Evidence is accumulating that insulin resistance may be the common etiological factor for the individual components of the Metabolic Syndrome, although there appear to be heterogeneity in the strength of the insulin resistance relationship with different components between, and even within populations' (Alberti and Zimmet 1998: 548). 
medical studies distinguishes between nominal and real definitions: according to von Zimmerman (1763), the former points to illness on the bases of clinical phenomena, whereas the latter reveals the true nature of the illnesses and is based on 'the knowledge of the relative causes' (see Federspil et al. 2006: 449). In modern medicine, the distinction is restated as the contraposition between extensive or nominal definitions, whose goal is to pick up the patients corresponding to a given category, and real definitions, which are intended to explain which is the object - the kind of disease - referred to by the name. Roughly, nominal definitions correspond to my type 1 definitions, as they denote just an association of pathological phenomena that occurs with a higher frequency than we would have expected if the phenomena under observation were independent (see Federspil et al. 2006: 452). Real definitions correspond to my type 3 definitions: 'When, on the other hand, the metabolic syndrome is defined by proposing a pathogenetic mechanism that explains the association between various illnesses, this means giving it a real causal definition' (ibid.)

Federspil et al. (2006: 449) claim that if the term 'metabolic syndrome' denotes merely the association of some metabolic disorders, then its utility is restricted to the identification of a certain category of patients. This would be exactly an example of a 'nominal' definition in contrast with 'real' definitions. According to these authors, nominal definitions are legitimate in clinical medicine, 'an applied science that sets out not only to discover, but also to organize incomplete knowledge for clearly practical purposes' (Federspil et al. 2006: 554). However, in the specific case of MetS, they point to some problems that put into question a too easy generalisation of the very utility of nominal definitions.

As their objections are closely related to those presented by other authors (such as Reaven and Kahn) that I will discuss later, I give here a general exposition of three main critical remarks about nominal or type 1 definitions of MetS:

(a) Concerning the case of MetS, the diagnostic utility of a mere list of predictive factors brings about what we could call an instability of reference, which is instability on the class of individuals to whom we can attribute the property 'affected by metabolic syndrome'. 
(b) If for 'practical utility' we mean not only 'predictive utility' but also utility for prevention, treatment, therapy, and public health care planning, then the list of factors must point out not only the individual factors but also the conjunction of the components. Therefore, the definition should find a justification in some kind of unifying treatment that is more efficacious than the treatment of the individual components (taken one by one).

(c) If such a requirement appears too onerous, we should at least require that a multi-factor definition such as the MetS has a practical utility to efficaciously remind doctors and patients which parameters should be monitored to prevent future diseases. However, this minimalistic and purely psychological rendering of the syndrome requires a justification that shows that the definition is more efficacious than other parameters and, at the same time, does not cut off relevant factors.

I will discuss the topic of practical utility in the next section; in what follows, I will try to give some examples of the negative consequences of the instability of reference.

As we have seen, many institutions or panels (WHO, EGIR, ATP III, and others) have proposed different definitions intended as diagnostic tools, mainly as sets of risk factors for T2D and CVD. However, the different definitions are unable to select the same factors as necessary and sufficient criteria to label patients as having MetS. If, on the other hand, we allow that just a subset of the properties characterising MetS are possessed by individuals, we run the risk of labelling as 'MetS patients' individuals much different from one another. It is worth quoting the analysis by Federspil et al. of the problem raised by the plurality of definitions of MetS:

If we consider all patients who present the various recognized traits of MetS, or if we represent them using Venn's diagrams, however many traits there may be, it can be seen that if only two traits are considered - for example type 2 diabetes and obesity - then there will be just one condition characterized by the presence of both traits. If the syndrome's traits are four and at least three the components needed in order to diagnose it, the association that allows diagnosis are five ... Now, if we consider that the various definitions of metabolic syndrome 
consist of at least ten pathological phenomena (hyperglycemia, hyperinsulinemia, insulin resistance, central obesity, hyperlipidemia, arterial hypertension, microalbuminuria, hyperuricemia, prothrombotic and prohinflammatory states), it is easy to understand that the possible clinical pictures of the condition are too numerous to be of much help in diagnosis or prognosis (Federspil et al. 2006: 453).

What Federspil et al. presented as an abstract hypothesis finds an empirical counterpart in an Australian study, called the AusDiab Study (see Alberti 2005: D5). Here the different prevalence rates for MetS by three of the different definitions referred to above (WHO, EGIR, ATP III) are represented with Venn diagrams. It is worth noting that not only do the three definitions select slightly different percentages of the population as being affected by MetS, but only a subpart of the diagnosed 'patients' meets the joint criteria for all three definitions. Moreover, the most external parts of the chart represent non-overlapping groups of 'patients'.

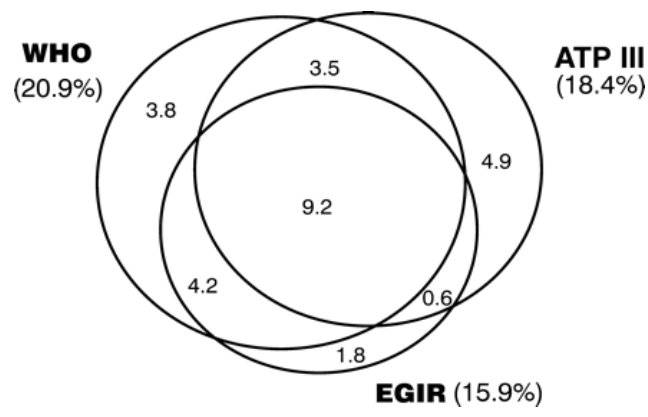

Figure 1: Percent prevalences within the total population (from Alberti 2005)

By criticising the purely nominal definitions of MetS proposed by various panels of experts, Federspil et al. (2006) advocate the search for a real definition. They find in the definition of Syndrome X proposed by Reaven (1988) a proper causal definition that appears very different from the descriptive definitions proposed by various panels of experts. The causal character of Reaven's definition makes it more reliable from both a clinical and a scientific point of view. The definition selects insulin resistance, but not abdominal obesity, as a necessary component of the syndrome and, at the same time, as the starting point for the construction of a pathophysiological mechanism 
that explains the syndrome. On the other hand, if we follow other definitions and take - as a necessary component of the syndromeabdominal obesity, but not insulin resistance, the syndrome should be considered as pointing to a different pathogenic mechanism, and therefore to a different disease (Federspil et al. 2006: 454).

Federspil et al. point out that Reaven's definition of Syndrome X is different from the definitions of panellists, because Syndrome $\mathrm{X}$ is endowed with causal import and is founded on a precise pathophysiological hypothesis. However, the association of a precise pathophysiological mechanism is not necessary for the attribution of casual import to a definition or for its being only a classificatory tool. Actually, a definition could be associated to a causal structure not grounded on a precise mechanism, but of a different kind, as the causal structures of graphical causal models. In this case, we would have a 'type 2 definition'. Even if, to my knowledge, nobody has presented a detailed graphical causal model (DAG-based) as an explicit definition of MetS in the period we are considering, I think this approach is worth discussing with respect to the definition of MetS. In fact, as we will see in the next section, on the one hand, Bayesian nets have been explicitly considered with respect to this topic; on the other, some of the problems linked to causal modelling - such as the relation between statistical data and back ground theoretical knowledge - play a crucial role in the discussion about MetS.

\subsection{Type 2 definitions: causal modelling}

It is worth remarking that, concerning the definition of MetS, the plurality of definitions and the consequent vagueness of the notion of MetS are considered not only a theoretical problem, but also and mainly a practical problem, casting doubts on the applicability of such a notion in diagnostic and therapeutic practices. The need for a more precise definition of MetS is strongly felt in the medical community, and many papers published at the beginning of the $21^{\text {st }}$ century testify to this. ${ }^{5}$ But which kind of definition is needed? Medical sciences

\footnotetext{
${ }^{5}$ Here are some: Leśniak and Kolasińska-Kloch 2003, Lim et al. 2004, Jørgensen and Borch-Johnsen 2004, Real and Carmena 2005, Rosas Peralta 2005, Eschwège 2005, Liberopoulos and Elisaf 2005, Aguilar-Salinas et al. 2005.
} 
do not strictly require definitions expressed as a set of necessary and sufficient conditions. Kincaid (2008) has noted that research on cancer makes progress notwithstanding the fact that there is no precise definition of the cancerous cell and that the expression 'cancer' is related to very different pathological phenomena. He also remarks that the difficulty of giving a precise definition of depression does not entail the non-existence of the pathology (Kincaid 2008: 375).

We are then back to the apparently weaker pragmatic stance according to which the definitions of panellists are of 'practical utility' (see the previous section at points (b) and (c)). But those definitions often reveal a hidden tension: on the one hand, they appear to be mere 'nominal' definitions linked only to practical utility; on the other hand, they hint at unknown causes underlying statistical associations and therefore seem to assume a non-specified underlying set of interacting causal factors. In what follows, I will try to explore this suggestion and see what result we may gain, if any.

The definitions given by panellists are basically disjunctions of conjunctions of properties, where each conjunction is associated to an increased probability of certain pathologies. Can we still speak of 'mechanisms' in this context? Well, yes and no. Type 3 definitions, such as Reaven's, aim to give mechanistic explanations of a (pathological) phenomenon. Here the term 'mechanism' is intended in the sense specified by recent research in mechanistic causation, by authors such as Machamer, Darden, Craver, Bechtel and Abrahamsen, and Glennan. This notion is efficaciously synthesised by Campaner:

Over and above differences between individual accounts, it can be claimed that a mechanism is usually construed as a system composed of entities that interact with each other. Entities must be localized and hierarchically organized within the system. It is not only their presence but also the organization of the entities and their activities that determine the ways in which they produce the phenomenon (Campaner 2011: 8).

It should be noted that mechanisms of this kind are often, if not always, multi-level. But we may also consider a more abstract notion of mechanism, where the 'mechanism' is conceived as a set of variables and a set of causal relations among variables on the same 
level'. 'Mechanisms' of such a kind are particularly suitable to be represented and studied using graphical causal models (Pearl 2000; Spirtes et al. 1993). Because the use of the term 'mechanism' to denote these kinds of causal structures can be misleading, ${ }^{7}$ I will just call them 'causal structures'. I will call any representation of causal structures a 'causal model'.

The point I want to make is that some of the panellists' definitions of MetS can be interpreted as definitions pointing to causal structures, either implicit or not fully defined. ${ }^{8}$ From this viewpoint, those definitions would not be merely associational definitions, but instead, they would a certain degree of causal import. Here, however, the kind of causality involved is based on statistical data and general principles of causal reasoning, and closer to the graphical modelling approach than to the strictly mechanistic one. However, in this case, many type II definitions could be criticized not only for their inability to select an appropriate class of patients (as it happens with nominal definitions), but also for being associated to a causal model that, as we shall see, does not adequately represent the real causal structure under investigation. In this perspective, most of the criticisms against the panellists' definitions can be interpreted as motivated by the inadequacy of the underlying causal models and by the lack of an open discussion on the building of such models.

The formal approaches of causal models elaborated by Spirtes, Pearl and many others offers a normative account of reasoning with causes and probabilities, providing principles for representing and

${ }^{6} \mathrm{H}$. Kincaid (2011: 73) distinguishes between vertical mechanisms, i.e. 'the component processes realizing some higher level capacities', and horizontal mechanisms, sets of 'intervening or mediating variables between a putative cause and its effect'. The latter are called 'horizontal' because the intervening or mediating variables are at the same level as the putative causal relata. My 'causal structures' are 'horizontal' in the sense specified by Kincaid. See also Kincaid 2012.

${ }^{7}$ Thanks to an anonymous referee for pointing this out.

${ }^{8}$ For example, Alberti et al. (2005: 1060) state that 'Central obesity, was agreed as essential [...] because of the strength of the evidence linking waist circumference with cardiovascular disease and the other metabolic syndrome components, and the likelihood that central obesity is an early step in the aetiological cascade leading to full metabolic Syndrome'. Here central obesity is seen as a cause of 'full MetS' due to statistical and chronological considerations. 
inferring causal relations. The causal structure of the phenomenon under investigation is represented by means of a causal diagram, a directed acyclic graph (DAG), in which nodes associated to variables are connected by arrows, where the direction of the arrows represents the direction of the causal relationship. A is a 'direct cause' of B if there is an arrow going from node A to node B. Causal diagrams assume an underlying causal structure; however, when a probability distribution is associated to a DAG, the graph can have both a causal and a probabilistic interpretation. The theory of causal modelling offers important directions for the selection of sets of possible causal structures from a set of statistical associations. However, as is well known, statistical data and DAG theory do not, per se, automatically reveal the causal structure of the domain, and the construction of causal models requires careful consideration of many other aspects, mainly the careful choice of the variables.

If we now review some of the main criticisms of the panellists' definitions of MetS, particularly ATP III, we may verify that those criticisms mainly concern the choice of the set of relevant variables, the dependence relations between them, and the inadequacy of the underlying causal models. Let us give a short summary of these three kinds of criticisms:

(i) Choice of variables

Shahar (2010) criticizes pannelists' definitions of MetS on the basis of methodological considerations. He distinguishes between natural variables (like height, weight, and fasting glucose levels) and derived variables (like body mass index, or 'diabetes status'). While natural variables have been created by nature and 'we just try to measure those values', artificial variables are human-made. We derive their value by means of arithmetical formulas, or conditional statements such as 'If fasting glucose is $<\mathrm{C}$, then [the value of] 'diabetes status' is 'no diabetes". (see Shahar 2010: 773) According to Shahar, in the pannellists' definitions 'MetS status' is a derived variable. He points out that they all conform to a standard format, represented by the following algorithm

Let $\mathrm{V}_{1}, \mathrm{~V}_{2}, \ldots, \mathrm{V}_{n}$, denote a set of $n$ continuous variables, either natural or derived. For each variable, decide on a cut-off point and derive a binary variable $(0,1)$ on the basis of that cut-off point and a conditional. Next, add 
up the values of these binary variable to derive a summation variable, say SUM. Finally, derive 'metabolic syndrome status' from SUM, using a cutoff point and a conditional: if SUM $<\mathrm{k}$, then the metabolic syndrome is absent; otherwise, the metabolic syndrome is present (Shahar 2010: 773).

Shahar emphasizes that definitions based on derived variables have limited epistemic import. Derived variables exist in the trivial sense that 'we create them from some other variables', but this does not implies that a derived variable has any empirical counterpart with genuine causal capacities. Furthermore, in the panellists' definitions the statistical associations among the derived variables remain unexplained, as the definitions do not mention any causal relation among derived variables or any common cause. Using DAGs, Shahar shows that definitions obtained according to the algorithm quoted above are too weak, as they are compatible with different causal structures and do not allow to exclude spurious causal relations.

Further criticisms raised by Shahar are shared by other authors. Overall, they focus on four weak points in the panellists' definitions concerning the choice of variables:

(a) Non-homogeneity-The various definitions of MetS refer to different sets of variables, as discussed above.

(b) Lack of upper cut-off limits — Since all of the syndrome variables have no upper cut-off limits, Kahn (2006) suggests that many individuals will be so diagnosed because they have frank diabetes, hypertension, or severe lipid abnormalities.

(c) Inappropriate dichotomising of variables - Reaven (2006) underlines how this aspect, together with the previous one, may have paradoxical consequences. Kahn (2006) is also concerned about how the label of MetS can be misleading to both the physician and the patient. ${ }^{9}$ The arbitrariness of the con-

\footnotetext{
${ }^{9}$ His rhetorical remark is worth quoting in full: 'Labelling a person with the metabolic syndrome can be very misleading to the physician and the patient. The syndrome is defined by dichotomizing continuous variables and providing no upper limits to any of them. Thus, a person who has an FPG of $105 \mathrm{mg} / \mathrm{dl}$ and a systolic blood pressure of $130 \mathrm{mmHg}$ and is only slightly overweight would be classified as having the syndrome. Meanwhile, another person with uncontrolled
} 
struction of binary variables is also stressed by Shahar (2010).

(d) Incompleteness of the model - Kahn et al. (2005) underline the arbitrary exclusion of some well-known risk factors for CVD, such as physical inactivity, family history, sex, and age.

(ii) Dependence relations between variables

Most criticisms are based on the claim that the panellists' definitions do not adequately match the causal relations among variables and, consequently, do not constitute a useful tool for diagnosis or treatment. The best example is given by the lack of a clear causal role played by obesity. I will discuss this aspect in section 3.2.

(iii) Causal inadequacy of the model

Given that the structure of causal relations of MetS is not defined or clear, we are left with a high degree of uncertainty as to which variables need to be intervened upon and whether the treatment of the syndrome must be something different from the individual treatment of the component variables. Common opinion is that at least some elements of MetS (e.g. dislipidaemia and hypertension) should be treated in any case. Perhaps, given the (causal) darkness of MetS, we should not be surprised that the only treatment actually shared from the various definitions seems to be the joint recommendation of a healthy diet and physical activity. We do not need MetS for that.

Eventually, a diagnosis of MetS according to the ATP III criteria could lead to neglecting a diagnosis of insulin resistance. In that case, the definition might lose even that minimal utility that was suggested at point (c) of paragraph 2.1. From a clinical point of view, such an approach increases the risk of leading to an over-evaluation of the harmful potential of some factors and to an under-evaluation of the danger of others. On the basis of these considerations, and of those expressed by ADA/EASD quoted above, the presumed pedagogical

diabetes, a systolic blood pressure of $165 \mathrm{mmHg}$, and morbid obesity would also be classified as having metabolic syndrome. Are both individuals at similar risk for a future myocardial infarction or stroke? What if the first person had an LDL cholesterol of $75 \mathrm{mg} / \mathrm{dl}$, was 40 years of age, was female, and had no family history of CVD?' (Kahn 2006: 1695). 
utility of MetS in promoting a healthier lifestyle also loses plausibility.

I tried to show how we can associate a causal content also to panellists' definitions of MetS, although the outlines of the associated causal models are not up to standards: they point to causal structures that do not work properly or are not well devised. Better awareness of how to build models could help avoid part of the confusion or vagueness linked to the definition of MetS. However, we may doubt whether the causal content of these models could have been sufficient.

The remaining part of this work is devoted to the third kind of definition, associated with a pathophysiological mechanism. I will also argue that Reaven's account of the syndrome is particularly well suited to a rational reconstruction in terms of natural kinds.

\section{Kinds of syndromes}

In what follows, I suggest that the differences of approach between 'the panellists' view' and Reaven's view can be partly clarified with the help of the philosophical research on natural kinds (see, for instance, Boyd 1991, 1999; Craver 2009). After a short survey of different kinds of 'natural kinds', I will direct attention back to Reaven's view and his criticism of the panellists' theories in order to defend the explicative value of a highly detailed causal definition of the syndrome.

\subsection{Kinds of kinds}

I will take the route suggested by Kendler et al. (2011). The authors start from the question 'What kinds of things are psychiatric disorders?' and examine four groupings of things, namely essentialist kinds, socially constructed kinds, practically constructed kinds, and mechanistic property clusters. As the partition proves very useful in reflecting on MetS, I will describe briefly the four kinds and assess their respective merits as candidates for rational reconstructions of MetS.

Essentialist kinds-Essentialist kinds are characterised by the fact that they exist independently of our minds and by having essences. Kendler et al. (2011: 1144) define essences as 'sets of features necessary and sufficient for something to count as a member of that kind and for which many identifying characteristics of that kind arise'. Essentialist kinds are therefore characterised by an emphasis on a 
single, well-defined aetiological agent, although this agent can be provisionally unknown.

If we look for 'these kinds of things' in medicine, we find that tuberculosis infection is a plausible candidate (due to its close link with the Mycobacterium Tuberculosis); another plausible candidate is Down syndrome, given that the trisomy of chromosome 21 is considered a necessary and sufficient condition for it. When we compare MetS with these diseases, it is apparent that neither in Reaven's nor in other definitions of MetS can we find the cause, intended as the sufficient and necessary condition, of CVD or T2D and other pathologies.

We could ask whether insulin resistance is 'the essence' of Reaven's Syndrome X. Actually, Syndrome X has, with insulin resistance, a unifying factor, which is a necessary condition for the syndrome, but insulin resistance it is not a sufficient condition, because, as we saw in section 1.1, it must be associated with other factors to produce an increased risk of CVD or T2D.

We can conclude that neither Syndrome X nor MetS is an essential kind. However, in Reaven's version, the syndrome is clearly considered an entity that exists independently of our descriptions and not a conventional construction. Moreover, the efforts to ascertain a 'unitary framework', in Reaven's words, or a 'real definition', in Federspil's words, show a certain 'closeness' with essentialist kinds.

Socially constructed kinds - According to a constructionist point of view, pathological kinds do not exist independently of the human mind, but they are the product of categorisation or classification by specific societies or by the prevailing culture in different historical periods. Given the ubiquity of the expression 'metabolic syndrome' in the media and its connections with different definitions (or no definition at all) and the contemporary lifestyle, it would be difficult to deny that the notion of MetS also has a cultural significance. However, it seems unlikely that an account of the syndrome in terms of social practices can actually cover the whole set of problems linked to the definition of MetS.

Practical kinds - Treating natural categories as practical kinds amounts to considering them as tools or instruments for the achievement of practical goals. Accordingly, they should be evaluated by their practical success, without asking if they are real or what are their true essences. With regard to MetS, at least some of the 
conventionalist approaches fit in this category. The clearest case is the WHO definition, in which the clustering anomalies are simply listed, and no criterion of importance or of strength of association is dictated. The underlying view of this definition is that controlling some well-known risk factors can prevent some major diseases, even if their causes remain unknown, and that it is important to provide physicians with a practical diagnostic tool. We have already commented on the shortcomings of this perspective in section 2.2.

Mechanistic property clusters - A different notion of natural kind is the notion of homeostatic property cluster, introduced by Boyd (1991) to describe biological species. Under this view kinds are not the product of human constructions or conventions; rather, their definition is specified a posteriori by a set or a cluster of properties whose possession is due to the causal structure of the external world (homeostatic property cluster ). The most important traits of homeostatic property clusters are the following:

(i) there is a family $\mathrm{F}$ of properties that co-occur in a relevant number of cases;

(ii) either the presence of some of the properties in $\mathrm{F}$ tends to be associated with the presence of the others, or there are underlying mechanisms or processes that tend to maintain both;

(iii) the conjoint occurrence of all, or many of the properties in question, together with the underlying mechanism(s) produces important effects;

(iv) 'imperfect' (incomplete) mechanisms are possible; and

(v) in many cases, the classifications as a specific property cluster will be indeterminate (Boyd 1999: 144).

An application of these ideas to medicine is suggested by Kendler et al (2011), who analyse psychiatric syndromes in terms of their property clusters, rather than in terms of their essences or as social constructs. They also propose to substitute the term 'homeostatic property cluster' with the more informative 'mechanistic property cluster'. I agree with them on this point, and from now on I will use their terminology. 
The definition of the syndrome proposed by Reaven in 1988, and its subsequent refinements, meets - at least partially — the defining criteria for mechanistic property clusters. The co-occurring phenotypic traits listed by the definition share the property of being parts of a unique pathophysiological mechanism. This mechanism is constituted by the totality of the causal influences exerted by insulin resistance on the other components and, in some cases, by the causal influences of some components on the others. The occurrence of the components of the syndrome and the underlying mechanism has the conjoint effect of increasing the risk of further pathologies. As quoted above, Reaven (1988: 1606) admits that not all the components of the syndrome must be present; moreover, as we have seen, the family of the components was enlarged in subsequent refinements of the original definition, as IR syndrome.

We have already analysed how some of the panellists' definitions were grounded on stable co-occurrences of properties that bring about important effects, and could therefore be associated to implicit causal models. The true difference between Reaven's approach and the panellists' approach lies in the fact that the former has a precise hypothesis about the causal mechanism underlying the syndrome. This hypothesis guides the criteria for inclusion or exclusion of variables that enter the definition and the choice of the most plausible causal relations between variables.

\subsection{Kinds of evidence}

According to our rough classification of definitions, Reaven's IR syndrome appears to imply a definition at a high level of causal specification. In the pathophysiological framework suggested by Reaven, everything is connected, even if not everything is explained; insulin resistance, the fundamental explicative factor, is not.

To explain the different links that connect the components of IR syndrome with related pathologies, Reaven always produces statistical evidence and, sometimes, mechanistic evidence. Both kinds 
of evidence are produced for T2D, ${ }^{10}$ Polycystic Ovary Syndrome, ${ }^{11}$ $\mathrm{CVD},{ }^{12}$ and non-alcoholic fatty liver disease. ${ }^{13}$

Only statistical evidence is produced for essential hypertension, different kinds of cancer, and obstructive sleep apnea. For instance, concerning the latter, Reaven refers to studies that seem to show that the frequency of obstructive sleep apnea among obese patients who are insulin resistant or hyperinsulinemic is greater than among equally obese patients without insulin resistance / hyperinsulinemia. Moreover, insulin resistance has been found in non-obese persons with obstructive sleep apnea. In this case, evidence is not conclusive, but the model is open:

This area of clinical investigation is just beginning, and it is premature to decide if obstructive sleep apnea is simply more likely to occur for mechanical reasons in obese individuals and insulin resistance represents an epi-

10 'Type 2 diabetes only occurs when insulin-resistant individuals are no longer able to maintain the degree of compensatory hyperinsulinemia needed to maintain normal glucose homeostasis' (Reaven 2004: 287).

11 'PCOS is another example in which adverse consequences of muscle and adipose tissue insulin resistance are caused by the associated hyperinsulinemia acting on normally insulin sensitive tissues In this instance, it seems that the clinical features of PCOS result from an increase in testosterone secretion by ovaries that are at least normally insulin sensitive, secondary to the higher insulin concentrations' (Reaven 2004: 289).

12 'Values of insulin-mediated glucose disposal vary continuously throughout a population of apparently healthy persons, and a difference of ${ }^{3} 600 \%$ exists between the most insulin-sensitive and the most insulin-resistant persons. Approximately $50 \%$ of this variability can be attributed to differences in adiposity (25\%) and fitness (25\%), with the remaining 50\% likely of genetic origin. The more insulin-resistant a person, the more likely that he or she will develop some degree of glucose intolerance, high triacylglycerol and low HDL concentrations, essential hypertension, and procoagulant and proinflammatory states, all of which increase the risk of cardiovascular disease' (Reaven 2006: 1237).

13 'Resistance to insulin action at the level of the muscle and adipose tissue results in daylong increases in circulating plasma insulin and free fatty acid concentrations, which lead to increased hepatic triglyceride synthesis. If the rate at which the liver is able to incorporate the newly synthesized triglyceride into very low density lipoprotein and secrete it as very low density lipoprotein-triglyceride lags behind the liver's synthesis of triglyceride, the result is an increase in hepatic fat content' (Reaven 2004: 289). 
phenomenon, or if insulin resistance/hyperinsulinemia may play a causal role in the genesis of sleep-disordered breathing (Reaven 2004: 291).

It is on the ground of mechanistic considerations that obesity is not included in Syndrome X or IR syndrome. As we have seen, abdominal obesity is present with different degrees of relevance and different denominations ('waist-to-hip ratio', 'waist circumference', 'central obesity') in many definitions of MetS, but it is absent in Reaven's definition. When Reaven proposed his Syndrome X, he was well aware that obesity was a risk factor for CVD and T2D; however, his exclusion of (abdominal) obesity 'was not an oversight' (Reaven 2011: 129). His account of the syndrome implied that the adiposity criteria included in the panellists' definition was qualitatively different from the other components, as the association of adiposity with the components is not direct, but rather due to the fact that excess adiposity increases the likelihood that a person will be insulin resistant (Reaven 2006: 1241).

In the same article, Reaven quotes a statistical study (Ninomiya et al. 2004) showing that abdominal adiposity is also not directly associated to CVD or stroke (and therefore its causal influence on these diseases should be mediated by other components of the syndrome). Obesity is a cause of insulin resistance, and its influence on both the components of Syndrome X and CVD is mediated by insulin resistance. For these reasons, it should be excluded from the cluster of factors composing Syndrome X or IR syndrome.

From an epistemic point of view, the characterisation of the syndrome as a mechanistic property cluster offers several advantages. Firstly, it allows one to see the mechanism underlying the syndrome as a component of a wider mechanism (for instance, of a mechanism including both adiposity, which is split from the components of Syndrome X but interacts with them) and the components of the syndrome. Secondly, it allows one to carefully distinguish the pathophysiological level of analysis from other levels, such as environmental stress, the gene level, and so on; a multilevel analysis aims to integrate different levels of explanation without obliterating the differences. 


\subsection{Different names, different concepts, and different goals?}

As it should now be clear, from a conceptual viewpoint the definition of Reaven's IR syndrome - with his previous Syndrome X-is deeply different from the definitions of MetS. In the already quoted essay 'The Metabolic Syndrome or the Insulin Resistance Syndrome? Different Names, Different Concepts, and Different Goals', Reaven (2004) underlines the difference between his approach and the approach of the panellists in defining MetS. His aim is to produce a conceptual framework in which to insert risk factors that are strongly associated with a set of clinical manifestations.

The primary goal of IR syndrome is therefore more explanatory than diagnostic. However, this pre-eminence of the explanatory goal does not imply that IR syndrome is useless from a clinical point of view, given that it may help to pinpoint 'mechanistic targets' with which to evaluate new treatments. MetS, on the contrary, is intended as a clinical tool and has to be evaluated in this respect. Reaven admits that, from this perspective, it could be reasonable to include obesity among the components of MetS; however, this choice is unsatisfactory because there is no general agreement on a shared and truly useful measure of obesity. Besides, as we have seen in section 2.1, the usefulness of MetS is strongly doubted because of its inability to identify and unambiguously select the class of 'patients' to which it should apply.

\section{Conclusions}

Federspil et al. (2006: 451) wrote: 'the debate about the definition of MetS continues at present, yet we believe that there is little hope of finding true agreement unless we stop to reflect on the basic concepts from a methodological point of view'. Ten years later, the controversy is still open, and recent work seems to confirm that the final explanation of the syndrome, if we ever will have one, will be extremely complicated, with intertwining mechanisms, loops, and ramifications. It is also plausible that the explanation will benefit from the contributions of massive amounts of clinical data, data regarding disease gene networks, human disease networks, and still others (e.g. see Kraja et al. 2008). 
My suggestion is that the recent advances in the theory of natural kinds can contribute to the methodological reflection, and, basically, that an analysis of the causal content of the different definitions may help to sort the range and limits of their application. Specifically, the controversy over MetS presents an illustration of how a unique tag has been applied to different definitions, built with different aims and with different principles, dealing with different levels of causality. These differences, however, do not imply a complete independence of the definitions.

First, our analysis suggests that type 1 definitions not only do not explain why certain people have a stronger risk of getting CVD or T2D, but also have a limited value in determining which people have the stronger risk.

Second, if we compare type 2 definitions (and causal models such as Bayesian Networks) with a mechanistic hypothesis like that of Reaven, we see that the latter has at least the heuristic capacity to guide the scientist's choice of the relevant variables and the detection of causal dependencies between them, narrowing the set of plausible DAGs.

As a conclusion, although different definitions may be used with different purposes, the hypothesis of a model with highly specified causal content such as a mechanistic property cluster, (that we have seen implemented in Reaven's work) may be used as a blueprint that helps clarifying the scope and limits of other kinds of definitions ${ }^{14}$.

Margherita Benzi DIGSPES

University of Eastern Piedmont Alessandria, Italy margherita.benzi@uniupo.it

${ }^{14}$ Previous versions of this paper have been discussed at a seminar at the Department of Science and Technology Studies of the University College (London 2014), and at the $6^{\text {th }}$ Philosophy of Medicine Roundtable (Bristol 2015) and at CaMits (Madrid 2015). I wish to thank participants in these events and in particular Brendan Clarke, Yafeng Shan, Jeremy Simon, Caterina Marchionni snd Julian Reiss for their suggestions. I also thank two anonymous referees for their abundant and useful comments. 


\section{References}

Aguilar-Salinas, C.A.; Rojas, R.; Gómez-Pérez, F.J.; Mehta, R.; Franco, A.; Olaiz, G.; and Rull, J.A. 2005. The metabolic syndrome: a concept hard to define Archives of Medical Research 36(3):223-31.

Alberti, K.G. 2005. Introduction to the metabolic syndrome. European Heart Journal Supplements 7 (D): D3-D5.

Alberti, K. G.; and Zimmet, P. F. 1998. Definition, diagnosis and classification of diabetes mellitus and its complications. Part 1: diagnosis and classification of diabetes mellitus. Provisional report of a WHO consultation. Diabetic Medicine 15(7): 539-53.

Alberti, K.G.; Eckel, P.; Grundy, S.M. et al. 2009. Harmonizing the metabolic syndrome. A joint interim statement of the International Diabetes Federation Task Force on Epidemiology and Prevention, National Heart, Lung, and Blood Institute; American Heart Association; World Heart Federation; International Atherosclerosis Society; and International Association for the Study of Obesity. Circulation 120: 1640-5.

Alberti, K. G. M.; Zimmet, P.; Shaw, J. and IDF Epidemiology Task Force Consensus Group. 2005. The metabolic syndrome - a new worldwide definition. The Lancet 366(9491): 1059-62.

ATP III. 2001. Executive summary of the third report of the National Cholesterol Education Program (NCEP) expert panel on detection, evaluation, and treatment of high blood cholesterol in adults (Adult Treatment Panel III). JAMA 285(19): 2486-7.

Balkau, B.; and Charles, M. A. 1999. Comment on the provisional report from the WHO consultation. Diabetic Medicine 16(5): 442-3.

Boyd, R. 1991. Anti-foundationalism and the enthusiasm for natural kinds. Philosophical Studies 61(1-2): 127-48.

Boyd, R. 1999. Homeostasis, species, and higher taxa. In Species: New Interdisciplinary Studies, ed. by R. Wilson. Cambridge, MA: MIT Press, 141-85.

Campaner, R. 2011. Understanding mechanisms in the health sciences. Theoretical Medicine and Bioethics. 32 (1): 5-17.

Craver, C. F. 2009. Mechanisms and natural kinds. Philosophical Psychology 22(5): 575-94.

Enzi, G.; Busetto, L.; Inelmen, E. M.; Coin, A.; and Sergi, G. 2003. Historical perspective: visceral obesity and related comorbidity in Joannes Baptista Morgagni's 'De Sedibus et Causis Morborum per Anatomen Indagata'. International Journal of Obesity 27(4): 534-5.

Eschwège, E. 2005. Metabolic syndrome: which definition(s) for which objective(s)?]. Annales d'Endocrinologie 66(2 Pt 2):1S32-44.

Federspil, G.; Nisoli, E.; and Vettor, R. 2006. A critical reflection on the definition of metabolic syndrome. Pharmacological Research 53: 449-56.

Fujioka, S.; Matsuzawa, Y.; Tokunaga, K.; and Tarui, S. 1987. Contribution of intra-abdominal fat accumulation to the impairment of glucose and lipid metabolism in human obesity. Metabolism 36: 54-9. 
Grundy, S. M.; Cleeman, J. I.; et al. 2006. Diagnosis and management of the metabolic syndrome. An American Heart Association/National Heart, Lung, and Blood Institute Scientific Statement. Current Opinion in Cardiology 21: 1-6.

Haller, H.; and Hanefeld, M. 1975. Synoptische Betrachtung Metabolischer Risikofaktoren. In Lipidstoffwechselstörungen, ed. by H. Haller, M. Hanefeld and W. Jaross. Jena: Gustav Fischer Verlag, 254-64.

Hitzenberger, K.; and Richter-Quittner, M. 1921. Ein Beitrag zum Stoffwechsel bei der vasculären Hypertonie. Wiener Archive für Innere Medizin 2: 189-216.

IDF (International Diabetes Federation). 2005. The IDF Consensus Worldwide Definition of the Metabolic Syndrome. Available at: www.idf.org/metabolicsyndrome.

Jørgensen, M.E.; and Borch-Johnsen, K. 2004. The metabolic syndrome-is one global definition possible? Diabetic Medicine 21(10):1064-5.

Kahn, R. 2006. The metabolic syndrome (emperor) wears no clothes. Diabetes Care 29(7): 1693-6.

Kahn, R.; Buse, J.; Ferrannini, E.; and Stern, M. 2005. The metabolic syndrome: time for a critical appraisal. Joint statement from the American Diabetes Association and the European Association for the Study of Diabetes. Diabetes Care 28(9): 2289-304.

Kendler, K. S.; Zachar, P.; and Craver, C. 2011. What kinds of things are psychiatric disorders? Psychological Medicine 41(06): 1143-50.

Kincaid, H. 2008. Do we need theory to study disease? Lessons from cancer research and their implications for mental illness. Perspectives in Biology and Medicine 51(3): 367-78.

Kincaid, H. 2011. Causal modelling, mechanisms, and probability in epidemiology. In Causality in the Sciences, ed. Phyllis McKay Illari, Federica Russo and Ion Williamson. Oxford: Oxford University Press: 70-90.

Kincaid, H. 2012. Mechanisms, causal modeling, and the limitations of traditional regression. In The Oxford Handbook of Philosophy of Social Science, ed. Harold Kincaid. Oxford: Oxford University Press: 46-64.

Kissebah, A.H.; Vydelingum, N.; Murray, R.; Evans, D. J.; Kalkhoff, R. K.; and Adams, P. W. 1982. Relation of body fat distribution to metabolic complications of obesity. The Journal of Clinical Endocrinology and Metabolism 54 (2): 254-60.

Kitano, H.; Oda, K.; Kimura, T.; Matsuoka, Y.; Csete, M.; Doyle, J.; and Muramatsu, M. 2004. Metabolic syndrome and robustness tradeoffs. Diabetes 53 (suppl 3): S6-S15.

Kraja, A. T.; Province, M. A.; Huang, P.; Jarvis, J. P.; Rice, T.; Cheverud, J. M.; and Rao, D. C. 2008. Trends in metabolic syndrome and gene networks in human and rodent models. Endocrine, Metabolic and Immune Disorders-Drug Targets 8(3): 198-207.

Kylin, E. 1921. Hypertonie und Zuckerkrankenheit. Zentralblatt für innere Medizin 42: 873-7.

Kylin, E. 1923. Studien über das Hypertonie-Hyperglykämie-Hyperurikämie Syndrom. Zentralblatt für innere Medizin 44: 105-27. 
Lam, D. W.; and LeRoith, D. 2015. Metabolic syndrome. In Endotext [Internet]. Ed. by L.J De De Groot, P. Beck-Peccoz, G. Chrousos et al. http://www. ncbi.nlm.nih.gov/pubmed/25905173.

Leśniak, W.; and Kolasińska-Kloch, W. 2003. Do syndromes X, cardiac and metabolic, have any similar characteristics?. Folia Medica Cracoviensia 44(1-2): 59-69.

Liberopoulos, E.N.; and Elisaf, M.S. 2005. Diagnosis of the metabolic syndrome: which definition should we use? The Hellenic Journal of Cardiology 46(4): 258-62.

Lim, H.S.; Patel, J.V.; Lip, G.Y. 2004. Metabolic syndrome: a definition in progress. Circulation 110(4):e35; author reply e35.

Marañon, G. 1922. Über Hypertonie und Zuckerkrankenheit. Zentralblatt für Innere Medizin 43: 169-76.

Mitka, M. 2005. Does the metabolic syndrome really exist? JAMA 294: 2010-3.

Ninomiya, J.K.; L'Italien, G.; Criqui, M.H.; Whyte, J.L.; Gamst, A.; Chen, R. 2004. Association of the metabolic syndrome with history of myocardial infarction and stroke in the third National Health and Nutrition Examination Survey. Circulation 109: 42-6.

Oda, E. 2012. Metabolic syndrome: its history, mechanisms, and limitations. Acta Diabetologica 49(2): 89-95.

Pearl, J. 2000. Causality: Models, Reasoning, and Inference. Cambridge: Cambridge University Press. $2^{\text {nd }}$ ed. 2010.

Real, J.T.; and Carmena, R. 2005. Relevance of metabolic syndrome and its definition depending on the criteria employed. Medicina Clinica 124(10): 376-8.

Reaven, G. M. 1988. Role of insulin resistance in human disease. Diabetes 37: 1595-607.

Reaven, G M. 2004. The metabolic syndrome or the insulin resistance syndrome? Different names, different concepts, and different goals. Endocrinology and Metabolism Clinics of North America 33(2): 283-303.

Reaven, G. M. 2005. The metabolic syndrome: requiescat in pace. Clinical Chemistry 51(6): 931-8.

Reaven, G. M. 2006. The metabolic syndrome: is this diagnosis necessary? The American Journal of Clinical Nutrition 83(6): 1237-47.

Reaven, G. M. 2011. The metabolic syndrome: time to get off the merry-goround? Journal of Internal Medicine 269 (2): 127-36.

Rosas Peralta, M. 2005. Definition of metabolic syndrome: Tower of Babel. Archivos de cardiología de México 75(2): 230-3.

Shahar, E. 2010. Metabolic syndrome? A critical look from the viewpoints of causal diagrams and statistics. Journal of Cardiovascular Medicine 11(10): 772 -9.

Spirtes, P.; Glymour, C. N.; and Scheines, R. 1993. Causation, Prediction, and Search. Lecture Notes in Statistics. $2^{\text {nd }}$ ed. Cambridge: Cambridge University Press, 2009.

Vague, J. 1947. La différenciation sexuelle: facteur déterminant des formes de l'obesité. Presse Medicale 30: 339-40.

Zimmermann, J. G. 1763. Von der Erfahrung in der Arzneykunst. 2 Bde. Zürich: bey Heidegger und Compagnie. 